\title{
Modified method for effective primary vascular smooth muscle progenitor cell culture from peripheral blood
}

\author{
Jin-Hee Seong $\cdot$ Yi-Sun Song $\cdot$ Hyun-Woo Joo $・$ In-Hwa Park $\cdot$ Guang-Yin Shen • \\ Na-Kyoung Shin $\cdot$ A-Hyeon Lee $\cdot$ Amy M. Kwon $\cdot$ Yonggu Lee $\cdot$ \\ Hyuck Kim $\cdot$ Kyung-Soo Kim (i)
}

Received: 10 February 2020/ Accepted: 2 September 2020/Published online: 9 September 2020

(C) The Author(s) 2020

\begin{abstract}
In previous studies, vascular smooth muscle progenitor cells (vSMPCs) isolated from peripheral blood mononuclear cells (PBMCs) were cultured using medium containing platelet-derived growth factor-BB (PDGF-BB) for 4 weeks. However, this method requires long culture periods of up to 4 weeks and yields low cell counts. Therefore, we proposed the modified method to improve the cell yield and purity and to reduce the cell culture period. PBMCs were isolated from human peripheral blood and cultured by the conventional method using medium containing PDGF-BB alone or the modified method using medium containing PDGF-BB, basic fibroblast growth factor (bFGF), and insulin-transferrin-selenium ITS for 4 weeks. The purity of vSMPCs was analyzed for the expression of a- smooth muscle actin (SMA) by flow cytometry and significantly higher in the
\end{abstract}

J.-H. Seong · Y.-S. Song · H.-W. Joo ·

I.-H. Park · N.-K. Shin · A.-H. Lee · K.-S. Kim

Graduate School of Biomedical Science and Engineering,

Hanyang University, Seoul, South Korea

\section{G.-Y. Shen · K.-S. Kim ( $\bowtie)$}

Division of Cardiology, Department of Internal Medicine, Hanyang University College of Medicine, Seoul, South

Korea

e-mail: kskim@hanyang.ac.kr

\section{G.-Y. Shen}

Division of Cardiology, Department of Internal Medicine, Jilin University Jilin Central Hospital, Jilin, China modified method than conventional methods at the 1 st and 2 nd weeks. Also, mRNA expression of a-SMA by real-time PCR was significantly higher in the modified method than conventional method at the 2 weeks. The yield of vSMPCs by trypan blue exclusion assay was significantly higher in the modified method than conventional method at the 1st, 2nd and 3rd weeks. The primary culture using the modified method with PDGF-BB, bFGF, and ITS not only improved cell purity and yield, but also shortened the culture period, compared to the conventional culture method for vSMPCs. The modified method will be a time-saving and useful tool in various studies related to vascular pathology.

Keywords VSMPCs - PBMCs - PDGF-BB · Peripheral blood . Cell culture

\footnotetext{
A. M. Kwon

Biostatistical Consulting and Research Laboratory, Medical Research Collaborating Center, Industry-

University Cooperation Foundation, Hanyang University, Seoul, South Korea

Y. Lee

Department of Internal Medicine, Hanyang University Guri Hospital, Guri, South Korea

H. Kim

Department of Thoracic Surgery, Hanyang University Seoul Hospital, Seoul, South Korea
} 


\begin{tabular}{ll}
\multicolumn{2}{l}{ Abbreviations } \\
$\alpha$-SMA & Alpha-smooth muscle actin \\
bFGF & Basic fibroblast growth factor \\
BM & Bone marrow \\
CD31 & Cluster of differentiation 31 \\
HASMCs & Human aortic smooth muscle cells \\
ITS & Insulin-transferrin-selenium \\
PBMCs & Peripheral blood mononuclear cells \\
PDGF- & Platelet-derived growth factor-BB \\
BB & \\
SMCs & Smooth muscle cells \\
smMHC & Smooth muscle myosin heavy chain \\
vSMPCs & Vascular smooth muscle progenitor cells
\end{tabular}

\section{Introduction}

Vascular smooth muscle progenitor cells (vSMPCs) are defined as circulating or resident cells localized within the bone marrow (BM), vascular wall, or perivascular area that are committed to the smooth muscle fate and capable of differentiating into mature smooth muscle cells (SMCs) (Merkulova-Rainon et al. 2012). Previous reports have demonstrated the role of bone marrow (BM)-derived smooth muscle progenitor cells in contributing towards the development and progression of atherosclerotic vascular diseases and neointima hyperplasia after vascular injuries (Han et al. 2001; Iwata et al. 2010; Sata et al. 2002; Shimizu et al. 2001; Zernecke et al. 2005; Zoll et al. 2008). vSMPCs were shown to be involved in the mechanism of plaque formation in an atherosclerotic animal model, as well as the process of neointimal formation after arterial injury.

Platelet-derived growth factor-BB (PDGF-BB) is involved in various physiological functions including blood vessel formation, vascular growth, and proliferation of SMCs. It has also been known to promote vSMPC differentiate into mature SMCs (Battegay et al. 1994; Hellstrom et al. 1999). Previous reports have described that vSMPCs could be cultured from peripheral blood mononuclear cells (PBMCs) in the medium containing PDGF-BB (Kang et al. 2014; Lin et al. 2016; Simper et al. 2002; Sugiyama et al. 2006; Xie et al. 2008). However, this conventional primary culture method requires at least a 4-week-long culture period and generally yields low cells counts (Wang et al. 2012). To use vSMPCs more efficiently in vascular disease research, it is desirable to develop a method for obtaining higher yields of vSMPCs in a shorter period of time than the conventional method using PDGF-BB containing medium.

Several growth factors and supplements have been introduced to improve the result of primary vascular SMC culture from the artery walls. Basic fibroblast growth factor (bFGF) is known to be involved in angiogenesis and to promote migration and proliferation of SMCs in the presence of PDGF-BB in the medium (Jackson and Reidy 1993; Kato et al. 1998; Millette et al. 2005). Supplementation of insulintransferrin-selenium (ITS) in the medium has also been reported to reduce the use of serum and promote cell proliferation during primary culture of aortic SMCs (Chua et al. 2005; Waldbillig and Pang 1992).

Therefore, supplementation of these factors to the culture medium during the primary culture of vSMPCs may improve the efficacy of primary SMC culture. Here, we propose a newly modified method that uses a culture medium containing PDGF-BB, bFGF and ITS for the primary culture of vSMPCs from PBMCs. The purity and abundance of the vSMPCs cultured by the modified method were compared with those cultured by the conventional method using medium containing PDGF-BB alone.

\section{Materials and methods}

Isolation of vascular smooth muscle progenitor cells from human peripheral blood

The study protocol was reviewed and approved by Institutional Review Board of Hanyang University Medical Center (IRB No.; HYUH201506005003). Adult peripheral blood samples were obtained from 2 healthy human volunteers (1 male, age 29; 1 female, age 25 ) after informed consent was obtained. $20 \mathrm{ml}$ of human peripheral blood were collected in sterile vacutainer tubes containing the anticoagulant EDTA (BD Vacutainer Systems, Roborough, Plymouth, UK). PBMCs were isolated from the buffy coat blood after density gradient centrifugation using Lymphoprep (STEMCELL Technologies Inc., Vancouver, Canada). After washing 3 times with phosphate buffered saline (PBS; Gibco, Grand Island, NY, U.S.), the PBMCs was cultured in dulbecco's modified 
eagle's medium (DMEM; Gibco, Grand Island, NY, USA) containing $20 \%$ fetal bovine serum (FBS; Gibco, Grand Island, NY, USA); $1 \% 100 \mathrm{U} / \mathrm{ml}$ penicillin and $100 \mathrm{mg} / \mathrm{ml}$ streptomycin (P.S; Gibco, Grand Island, NY, USA); 50 ng/ml PDGF-BB (R\&D systems Inc., Minneapolis, Minnesota, U.S.) (Conventional method) or DMEM containing 20\% FBS; $1 \%$ P.S; 5 ng/ml PDGF-BB; 10 ng/ml bFGF (Biobud, Seongnam-si, Gyeonggi-do, Republic of Korea); ITS (10 ng/ml insulin-5.5 ng/ml Transferrin-5 ng/ml Selenium; Gibco, Grand Island, NY, U.S.) (Modified method) and placed on 6 well plate coated with type I collagen (Corning, Corning, New York, U.S.) at a density of $2 \times 106 /$ well. The cells were maintained in a humidified $37{ }^{\circ} \mathrm{C}$ incubator with $5 \% \mathrm{CO} 2$. The medium was replaced every 5 days. PBMCs was seeded at the 1 week intervals for 4 weeks (Fig. 1).

Fluorescence-activated cell sorting (FACS) analysis

The FACS analysis was performed to identify the $\alpha$ smooth muscle actin (SMA) expression in the vSMPCs cultured in both methods at 1st, 2nd, 3rd, and 4th week of experiment. Cells were harvested with StemPro accutase cell dissociation reagent (Gibco, Grand Island, NY, U.S.) and resuspended in 10\% FBS/ $0.1 \%$ sodium azide in PBS. Cells were fixed in Methanol:Acetone $=1: 1$ for $10 \mathrm{~min}$, intracellular antibodies permeabilized with $0.1 \%$ TritonT-100 (Sigma-aldrich, St. Louis, Missouri, U.S.) in PBS. The cells were incubated for 30 min with the primary antibodies to Alexa488-conjugaed human $\alpha$ - SMA (Abcam, Cambridge, UK) in 3\% bovine serum albumin (Sigma-aldrich, St. Louis, Missouri, U.S.) in PBS. After washing in $10 \% \mathrm{FBS} / 0.1 \%$ sodium azide (Sigma-aldrich, St. Louis, Missouri, U.S.) with PBS 3 times, analysis was performed using a FACS Canto flow cytometer (BD Biosciences, Franklin Lakes, New Jersey, U.S.). Data were analyzed using FACSDIVA software (BD Biosciences, San jose, California, U.S.). The experiments were repeated three times.

RNA isolation and quantitative real-time polymerase chain reaction (qRT-PCR)

qRT-PCR was performed to identify the amount of expression of a-SMA mRNA indicating the abundance of SMPCs cultured in both methods at 2 weeks of experiment. Total RNA was extracted from vSMPCs by using the Qiazol reagent (Qiagen, Hilden, Germany) following the manufacturer's instructions. RNA concentrations were measured with a Nanodrop ND-2000 spectrophotometer (Thermo Fisher Scientific Inc., Waltham, Massachusetts, U.S.), and purity was determined by measuring ratios of A260 and A280, which ranged from 1.8 to 2.0.

For qRT-PCR, complementary DNA (cDNA) was synthesized from 3 ug of RNA using Moloney Murine Leukemia virus reverse transcriptase primed with oligo (dT) (Invitrogen, California, U.S.). The qRTPCR was performed using a Light Cycler 480 System (Roche, Basel, Switzerland) with a FastStart DNA Master SYBR Green I kit (Roche Diagnostics, Indianapolis, U.S.). qPCR amplification was performed with an initial incubation for $10 \mathrm{~min}$ at $95^{\circ} \mathrm{C}$ followed by 45 cycles of $10 \mathrm{~s}$ at $95^{\circ} \mathrm{C}, 10 \mathrm{~s}$ at $60{ }^{\circ} \mathrm{C}$, and $8 \mathrm{~s}$ at $72{ }^{\circ} \mathrm{C}$ and then a final dissociation step at $65{ }^{\circ} \mathrm{C}$ for $15 \mathrm{~s}$. The crossing point ( $\mathrm{Cp})$ of each sample was automatically determined by the LightCycler program. The primers for human $\alpha$-smooth muscle actin (SMA) were forward 5'-CCGAGATCTCACTG ACTACCTCATG- $3^{\prime}$, reverse 5'-ACAATCTCAC GCTCAGCAGTAGTAA-3'. The primers for human glyceraldehyde 3-phosphate dehydrogenase (GAPDH) were forward 5'-GGTATGACAACGAA TTTGGCTACAG- $3^{\prime}$, reverse 5'-TCTCTCTCTTCC TCTTGTGCTCTTG-3' ${ }^{\prime}$. PCR was performed in duplicate and the measured transcript levels were

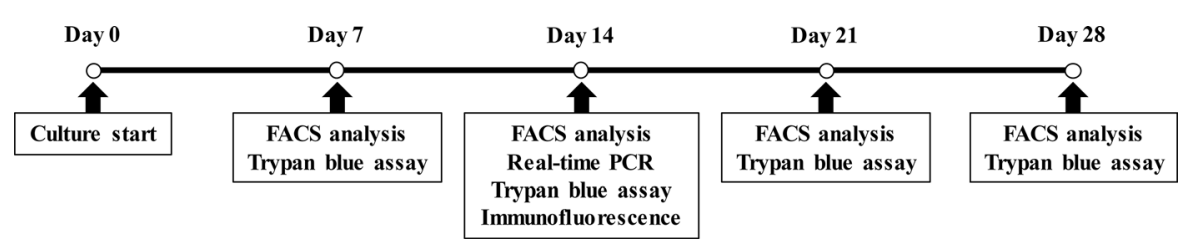

Fig. 1 Experimental scheme of vSMPCs culture. vSMPCs was cultured in the modified method and the conventional method for 4 weeks at 1 week of intervals, respectively 
normalized against those of GAPDH. The experiments were repeated three times.

Measurement of cell yield

Trypan blue exclusion assay was performed to determine the yield of vSMPCs in both methods at 1st, 2nd, $3 \mathrm{rd}$, and 4th week of experiment. Cells were harvested with StemPro accutase cell dissociation reagent and resuspended in PBS. Cell suspension was stained by $0.4 \%$ trypan blue dye (Gibco, Grand Island, NY, U.S.). Trypan blue-negative cells was counted with a hemocytometer (Paul Marienfeld GmbH \& Co. KG, Am Wöllerspfad, Lauda-Königshofen, Germany) under an inverted light microscope (XDS-3LT, OPTIKA Italy, Via Rigla, Ponteranica BG, Italy) at $100 \times$ magnification. The vSMPCs yield was calculated by multiplying the number of harvested cells to the percentage of a-SMA positive cells at 1,2, 3, and 4 weeks and dividing the value by the number of PBMCs initially. The experiments were repeated three times.

$$
\begin{aligned}
\text { Cell yield }(\%)= & (\text { Number of harvested cells } \\
& \times \alpha-\text { SMA positive cells } \%) \\
& /(\text { Initially number of PBMCs }) .
\end{aligned}
$$

Immunofluorescence (IF) staining

Immunofluorescence staining was performed to confirm the expression of $\alpha$-SMA and smooth muscle myosin heavy chain (smMHC) indicating the SMCs phenotype in SMPCs cultured in both methods at 2 weeks of experiment. Cells were fixed in methanol:acetone $=1: 1$ for $10 \mathrm{~min}$, permeabilized in $0.1 \%$ TritonX-100 for 5 min. Primary antibodies were used against $\alpha$-SMA, smMHC, cluster of differentiation 31 (CD31) (Abcam, Cambridge, UK). $\alpha$-SMA and CD31 was blocked in 10\% normal donkey serum (Vector Laboratories Inc., Burlingame, California, U.S.) in PBS. smMHC was blocked in $10 \%$ normal goat serum (Abcam, Cambridge, UK) in PBS. The cells were incubated at $4{ }^{\circ} \mathrm{C}$ with the primary antibodies to mouse anti- $\alpha$-SMA, rabbit anti-smMHC, and mouse anti-CD31. After washing in PBS, the cells were incubated for $1 \mathrm{~h}$ at room temperature with the secondary antibodies. Nuclei were stained with $4^{\prime}, 6-$ diamidino-2-phenylindole (DAPI; Vector Laboratories Inc., Burlingame, California, U.S.)
Immunofluorescent image captured using an inverted fluorescence microscope (LeicaDMI4000B, Wetzlar, Germany) at $200 \times$ magnification. Fluorescence-positive cells were counted in order to calculate the marker expression (number of fluorescence-labeled cells/number of total nuclei) in randomly 4 images in each group. The experiments were repeated three times.

\section{Statistical analysis}

We performed Kruskal-Wallis tests to examine whether there are significant differences of rank-sum in $\alpha$-SMA positive cell $(\%)$ and cell yields by the two different methods at four different culturing periods at $\alpha=0.05$ to avoid normal assumption due to sample size.Besides, we tested if there is a significant difference in mRNA expression levels and quantitative fluorescence analysis at the 2 nd week according to Kruskal-Wallis tests. All statistical methods were conducted using SAS 9.4 (SAS institute Inc. NC, USA).

\section{Results}

Purity of vascular smooth muscle progenitor cells according to culture period

In order to compare the purity of vSMPCs according to culture period, the percentage of $\alpha$-SMA positive cells was analyzed by FACS (Fig. 2 and Table 1). Population of $\alpha$-SMA positive cells was significantly different between the conventional method and the modified method $(P=0.0353)$. The purity of vSMPCs was significantly higher in the modified method than that in the conventional method at 1 week $(P=0.0495)$ and 2 weeks of culture $(P=0.0495)$. The purity of vSMPCs was the highest at 2 weeks of culture in both culture method; however, the purity rapidly reached $77.7 \%$ at 1 week of culture and remained stable after 2 weeks of culture in the modified method. The purity slowly reached $44.25 \%$ at 1 week of culture and was peaked after 2 weeks of culture in the conventional method. Cell purity did not significantly different between the vSMPCs at 2 weeks of culture in both culture method and in human aortic smooth muscle cells (HASMCs) as a positive control. (Modified 
method vs. HASMCs, $\mathrm{P}=0.513$; Conventional method vs. HASMCs, $P=0.127$ ).

mRNA expression of $\alpha$-smooth muscle actin

The expression of $\alpha$-SMA mRNA in vSMPCs cultured at 2 weeks was confirmed by real-time PCR (Fig. 3 and Table 2). The expression of $\alpha$-SMA mRNA was significantly higher in the modified method than that in the conventional method $(P=0.0495)$. And, expression of $\alpha$-SMA mRNA did not significantly different between in the modified method and in HASMCs as a positive control $(P=0.345)$, whereas was significantly lower in the conventional method than that in HASMCs $(P=0.046)$.

Cell yield according to culture period

In order to compare the cell yield according to culture period, cell number was performed by trypan blue exclusion assay (Fig. 4 and Table 3). The cell yield was significantly different between the conventional method and the modified method $(P=0.0179)$. The cell yield was significantly higher in the modified method than that in the conventional method at 1 week $(P=0.0495), 2$ weeks $(P=0.0495)$ and 3 weeks of culture $(P=0.495)$. The yield of cells peaked at 2 weeks of culture, then gradually decreased afterwards in both culture methods.

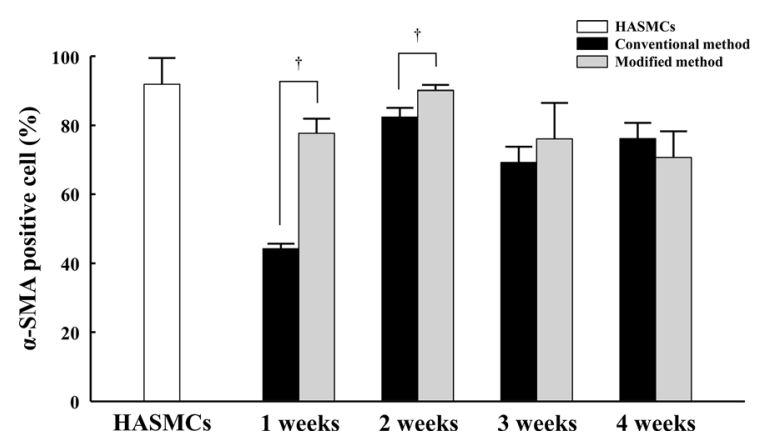

Fig. 2 The purity of vSMPCs measured with $\alpha$-SMA expression by FACS analysis. $\alpha$-SMA positive cell in HASMCs were measured as a positive control. All data are expressed as mean \pm SD. $\uparrow P<0.05$, Modified method vs. Conventional method. The experiments were performed in triplicate
Morphologic appearance of vascular smooth muscle progenitor cells

To confirm the cell morphologic appearance, vSMPCs were observed by microscopy. vSMPCs cultured in both culture methods at 2 weeks were appeared to be spindle-shaped cells with hill and valley pattern as a smooth muscle cell's morphology (Fig. 5a, b). To additionally evaluate phenotype, cells was stained using smooth muscle cell specific antibodies. The vSMPCs cultured in both methods at 2 weeks were observed expression of smooth muscle cells specific marker $\alpha$-SMA (Fig. 5c, d) and smMHC (Fig. 5e, f) by immunofluorescence staining. In the quantitative analysis results, the percentage of $\alpha$-SMA and smMHC positive cells did not significantly different between in the two method at 2 weeks $(\alpha-S M A$, $P=0.770$; smMHC, $P=0.905$ ) (Fig. 5i, j). Endothelial markers such as CD31 were stained to confirm the presence of endothelial cells and mononuclear cells in the cultured cells. However, expression of the endothelial cell surface marker CD31 was not observed in vSMPCs cultured in both methods at 2 weeks (Fig. 5g, h).

\section{Discussion}

In this study, the purity and abundance of vSMPCs were compared between the conventional culture method and the modified culture method, using the medium containing PDGF-BB alone and one containing a combination of PDGF-BB, bFGF, and ITS, respectively. The vSMPCs cultured in the modified medium showed higher purity and abundance than those cultured in the conventional medium at 2 weeks of culture. The purity of vSMPCs increased more rapidly from the 1st week of culture and remained steady after 2 weeks of culture under the modified medium, compared with that under the conventional medium. The yield of vSMPCs in the modified method was determined to be approximately twice that of the conventional method at 2 weeks of culture.

vSMPCs have been identified in the circulation, bone-marrow, and vascular walls (Majesky et al. 2011; van Oostrom et al. 2009). Circulating vSMPCs were originally inferred from animal studies that demonstrated the possible recruitment of circulating BMderived cells to sites of arterial remodeling and the 
Table 1 Kruskal-Wallis test $(\alpha$-SMA positive cell $(\%))$

$\chi^{2} ;$ Test statistic, $* * *$ p-value $<0.05$

\begin{tabular}{lll}
\hline Methods & $\chi^{2}$ & $P$ value \\
\hline Conventional vs. Modified & 4.45 & $0.0353^{* * *}$ \\
Conventional vs. Modified at the 1st week & 3.8571 & $0.0495^{* * *}$ \\
Conventional vs. Modified at the 2nd week & 3.8571 & $0.0495^{* * *}$ \\
Conventional vs. Modified at the 3rd week & 0.7843 & 0.3758 \\
Conventional vs. Modified at the 4th week & 1.1905 & 0.2752 \\
\hline
\end{tabular}

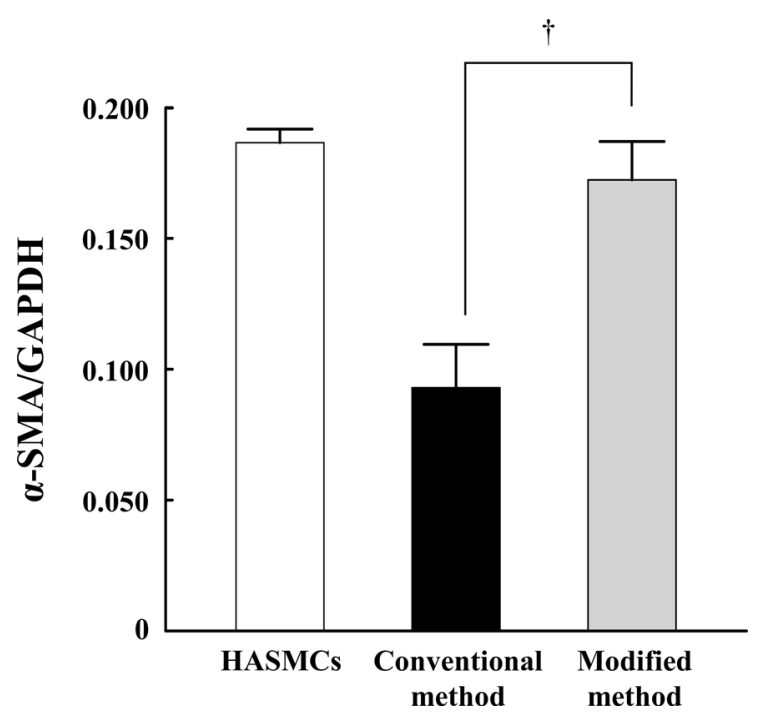

Fig. 3 mRNA expression of $\alpha$-SMA in vSMPCs at 2 weeks measured by real-time PCR. mRNA expression of $\alpha$-SMA in HASMCs was measured as a positive control. The transcripts levels were normalized by comparison with GAPDH. All data are expressed as mean $\pm \mathrm{SD}$. $\dagger P<0.05$, Conventional method $v s$. Modified method. The experiments were performed in triplicate

Table 2 Kruskal-Wallis Test (mRNA expression)

\begin{tabular}{lll}
\hline Methods & $\chi^{2}$ & P-value \\
\hline Conventional vs. Modified & 3.8571 & $0.0495^{* * *}$ \\
\hline$\chi^{2}$; Test statistic value, $* * *$ p-value $<0.05$ &
\end{tabular}

ability of haematopoietic stem cells to differentiate, in vitro and in vivo, into SMCs (Merkulova-Rainon et al. 2012; Sata et al. 2002; Shimizu et al. 2001). vSMPCs in mouse models stimulate collagen synthesis and reduce infiltration of inflammatory cells, thereby promoting a stable phenotype during the development and progression of atherosclerosis (Zoll et al. 2008). A distinct lineage of neointimal cells

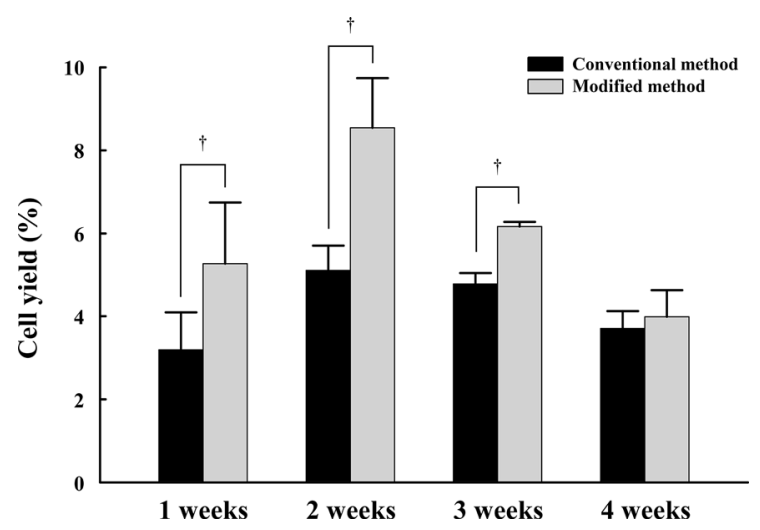

Fig. 4 The yield of vSMPCs assessed by trypan blue exclusion assay. All data are expressed as mean $\pm \mathrm{SD}$. $\dagger P<0.05$, Modified method vs. Conventional method. The experiments were performed in triplicate

derived from circulating bone marrow-derived cells, resembling immature vascular SMCs, is recruited during vascular repair after major vessel damage (Han et al. 2001). The expression of SDF- $1 \alpha$ has been implicated in neointima formation by mediating mobilization and recruitment of vSMPCs from peripheral blood while the levels of SDF- $1 \alpha$ are known to be differentially modulated in response to apoptosis after arterial injury (Schober et al. 2004???; Zernecke et al. 2005). In addition, recent studies have reported that the number of vSMPCs is higher in patients with vascular disease than in healthy individuals. Sugiuama et al. reported the presence of higher number of circulating cells expressing CD14-CD105 and a-SMA from peripheral blood in the coronary artery disease (CAD) patients compared to that in non-CAD patients (Sugiyama et al. 2006). These studies suggest that vSMPCs is closely related to the pathogenesis and repair mechanisms of various vascular diseases and injuries, implicating their wide utility in research studies associated with the field.

PDGF-BB is well-known for regulating blood vessel formation and vascular growth and promoting 
Table 3 Kruskal-Wallis test (yield)

$\chi^{2}$; Test statistic, $* * *$ p-value $<0.05$

\begin{tabular}{lll}
\hline Methods & $\chi^{2}$ & P-value \\
\hline Conventional vs. Modified & 5.6033 & $0.0179 * * *$ \\
Conventional vs. Modified at the 1st week & 3.8571 & $0.0495^{* * *}$ \\
Conventional vs. Modified at the 2nd week & 3.8571 & $0.0495^{* * *}$ \\
Conventional vs. Modified at the 3rd week & 3.8571 & $0.0495^{* * *}$ \\
Conventional vs. Modified at the 4th week & 1.1905 & 0.2752 \\
\hline
\end{tabular}
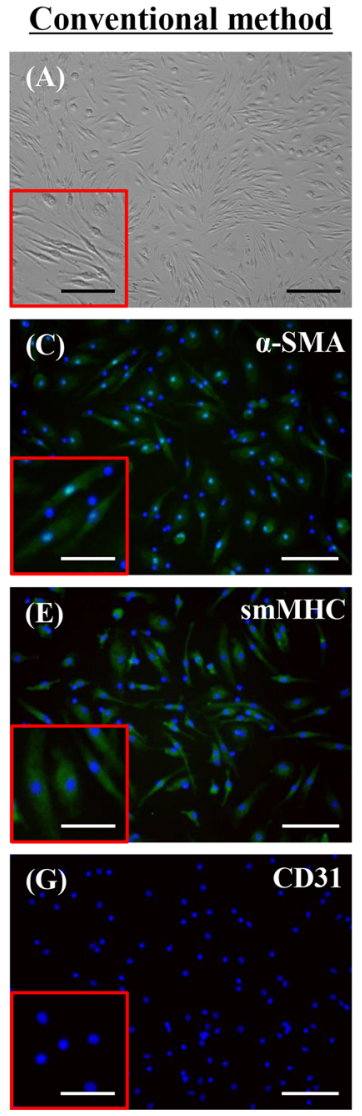

\section{Modified method}
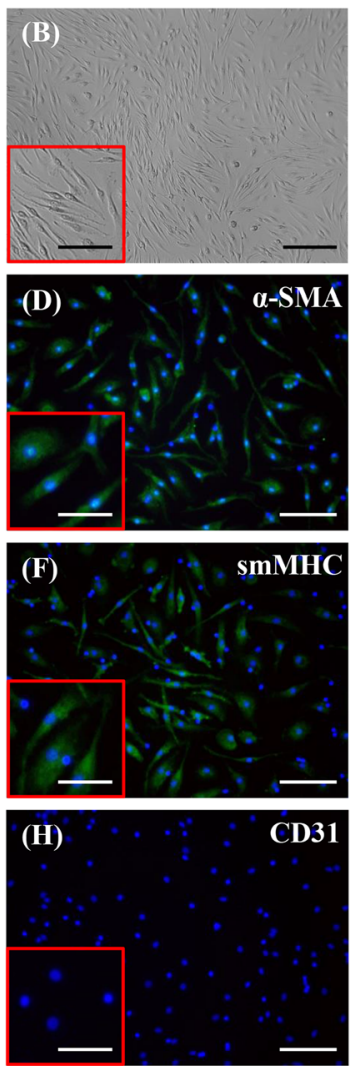

(I)

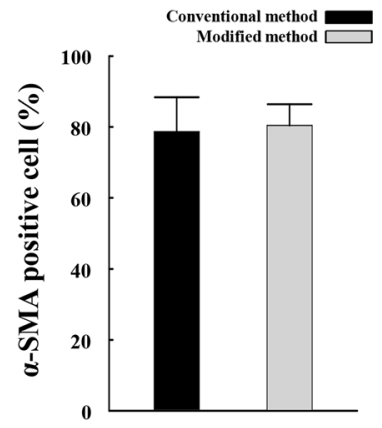

(J)

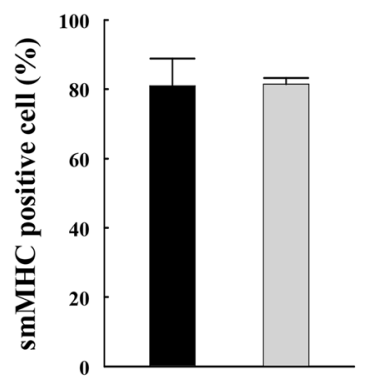

Fig. 5 Morphologic appearance and phenotype of vSMPCs observed by microscope. (a, b) Hill-and-valley morphology. (c, d) $\alpha$-SMA, (e, f) smMHC, (g, h) CD31 of immunofluorescence (green). (i, j) Result of quantitative analysis of smooth muscle cells specific marker $\alpha$-SMA and smMHC. CD31 of endothelial

SMC proliferation and migration (Battegay et al. 1994; Hellstrom et al. 1999; Jawien et al. 1992; Xie et al. 2014). Zhao et al. have reported that PDGF-BB promotes human pulmonary arterial SMC proliferation and survival (Zhao et al. 2014). PDGF-BB has also been known to stimulate the differentiation of vSMPCs into SMCs (Lin et al. 2016; Simper et al. 2002; Wang et al. 2016). In light of these findings, surface markers were stained as a negative control. Nuclei were stained with 4',6-diamidino-2-phenylindole (DAPI) (blue). Scale bar: $200 \mu \mathrm{m}(\mathbf{a}, \mathbf{b}) ; 100 \mu \mathrm{m}(\mathbf{c}-\mathbf{h}$, in the insets of $\mathbf{a}, \mathbf{b})$; $50 \mu \mathrm{m}$ (in the insets of $\mathbf{c}-\mathbf{h}$ ). The experiments were performed in triplicate

PDGF-BB has been used as the growth factor of choice in VSMPCs primary cultures. Thus, the conventional method for vSMPCs primary culture form peripheral blood employed the culture medium containing PDGF-BB alone as growth factors.

A long period for primary culture may be an important setback for researchers employing the conventional culture method. Primary culture for 
vSMPC conventionally requires long culture period of up to 4 weeks, as reported in previous studies. Simper et al. reported that only six to eight vascular progenitor colonies appear from total PBMCs after 3 weeks in culture with medium containing PDGF-BB (Simper et al. 2002). Kang et al. reported that vSMPC morphology begins to appear for about 4 weeks with medium containing PDGF-BB (Kang et al. 2014). Although these studies established the significance of PDGF-BB as a critical factor for vSMPC culture, they did not assess the efficacy of culture throughout the culture periods. In this study, we assessed the purity and yield on a weekly basis and, surprisingly, found that the purity and yield of vSMPCs peaked at 2 weeks, rather than at 4 weeks of culture, as described in the previous studies. This finding may thus provide many researchers ample scope to shorten research periods significantly.

Previous studies employing the conventional medium containing $50 \mathrm{ng}$ of PDGF-BB, which is 10 time higher than that in the modified medium (Joo et al. 2014; Lin et al. 2016; Simper et al. 2002). Our pilot studies have shown no morphological differences in vSMPCs cultured in the modified method when the concentration of PDGF-BB was $50 \mathrm{ng}$ and $5 \mathrm{ng}$.

Wang et al. reported that the yield of vSMPCs cultured by the conventional method using medium containing PDGF-BB was lower (Wang et al. 2012). In our study, the yield of vSMPCs in presence of the conventional medium at 2 weeks was low (about 5\%), whereas the yield of vSMPCs reached more than $8 \%$ in the modified method at 2 weeks of culture.

bFGF is a growth factor and signaling protein and mediates SMC migration (Chen et al. 2016b; Kato et al. 1998; Schroder et al. 2007). Several studies have reported that bFGF has the most robust effect on the ability of SMCs to induce a contractile to proliferative phenotype switch, in addition to controlling their growth and proliferation (Lindner and Reidy 1991; Shi and Chen 2014). Induction of bFGF signaling in SMCs inhibits TGF $\beta$ signaling and converts contractile SMCs to the proliferative phenotype (Chen et al. 2016a). PDGF-BB induces the release of bFGF and proliferation of human SMCs (Millette et al. 2005). Jackson et al. suggested that bFGF plays an important role in stimulating SMC migration and potentiates the movement of SMCs into the intima (Jackson and Reidy 1993). In the present study, it was anticipated that supplementation of bFGF promotes vSMPCs proliferation and provides a higher yield in the modified culture medium than in the conventional culture medium.

ITS is a medium supplement that reduces the use of serum and promotes the proliferation of many cells, including SMCs (Libby and O'Brien 1983). Chua et al. demonstrated the benefits of ITS supplementation in human chondrocytes monolayer culture (Chua et al. 2005). Lua et al. have shown that ITS may be permissive for sustained differentiation of embryonic cardiac muscle cells (Lau 1993). ITS supplemented medium in the aortic SMCs enhanced survival and prevents protein loss (Libby and O'Brien 1983). Thus, the role of ITS in the modified vSMPC culture medium may have been to sustain their differentiation into SMCs by promoting protein synthesis to express the SMC phenotype. Our results showed that vSMPCs in the modified culture medium containing PDGF-BB, bFGF, and ITS, were morphologically characterized as smooth muscle cells expressing their specific markers such as $\alpha$-SMA and smMHC.

Although this study paves the way for rapid culturing of vSMPCs through PBMC primary culture, we also acknowledge certain limitations. First, the related mechanisms by which PDDG-BB, bFGF, and ITS reduced the culture period and increased the yield in the culture of vSMPCs have not been delineated. Second, the optimal concentrations for PDGF-BB, bFGF, and ITS in the modified medium are yet to be established. Further investigation is needed to decide optimal concentrations of PDGF-BB, bFGF, and ITS in the culture of vSMPCs. Third, it remains to be elucidated as to what caused the high purity and yields of vSMPCs at 2 weeks in the conventional method in this study, unlike in the previous studies in which the purity and yields peaked at 4 weeks of culture. Finally, it is necessary to study a large cohort of human peripheral blood samples in the future. The number of sample for each group was small. Therefore, future studies should involve large number of sample.

Nevertheless, in conclusion, our results suggest that our modified method for vSMPC culture from peripheral blood using a medium supplemented with PDGF$\mathrm{BB}, \mathrm{bFGF}$, and ITS could provide higher purity and better yield of vSMPCs than the conventional culture method. In addition, the modified method shortened the culture period for vSMPCs. Overall, our improved culture method may provide a valuable resource to researchers in the field of cardiovascular diseases, 
especially arterial injury and repair, for conducting experiments more efficiently with vSMPCs derived from the peripheral blood.

Acknowledgments This research was supported by a grant of the Korea Health Technology R\&D Project through the Korea Health Industry Development Institute (KHIDI), funded by the Ministry of Health \& Welfare, Republic of Korea (Grant Number: HI16C0412).

Authors' contributions Conception or design: JHS, KSK Acquisition, analysis, or interpretation of data: JHS, SYS, HWJ, IHP, GYS, NKS, AHL Statistical Analysis: AMK Drafting the work or revising: JHS, YSS, HWJ, IHP, YGL, HK, KSK Final approval of the manuscript: KSK.

\section{Compliance with ethical standards}

Conflict of interest The authors declare that they have no conflict of interest..

Open Access This article is licensed under a Creative Commons Attribution 4.0 International License, which permits use, sharing, adaptation, distribution and reproduction in any medium or format, as long as you give appropriate credit to the original author(s) and the source, provide a link to the Creative Commons licence, and indicate if changes were made. The images or other third party material in this article are included in the article's Creative Commons licence, unless indicated otherwise in a credit line to the material. If material is not included in the article's Creative Commons licence and your intended use is not permitted by statutory regulation or exceeds the permitted use, you will need to obtain permission directly from the copyright holder. To view a copy of this licence, visit http://creativecommons.org/licenses/by/4.0/.

\section{References}

Battegay EJ, Rupp J, Iruela-Arispe L, Sage EH, Pech M (1994) PDGF-BB modulates endothelial proliferation and angiogenesis in vitro via PDGF beta-receptors. J Cell Biol 125:917-928. https://doi.org/10.1083/jcb.125.4.917

Chen PY, Qin L, Li G, Tellides G, Simons M (2016a) Fibroblast growth factor (FGF) signaling regulates transforming growth factor beta (TGFbeta)-dependent smooth muscle cell phenotype modulation. Sci Rep 6:33407. https://doi. org/10.1038/srep33407

Chen PY, Qin LF, Li GX, Tellides G, Simons M (2016b) Fibroblast growth factor (FGF) signaling regulates transforming growth factor beta (TGF beta)-dependent smooth muscle cell phenotype modulation Sci Rep-Uk 6 doi:ARTN 3340710.1038/srep33407

Chua KH, Aminuddin BS, Fuzina NH, Ruszymah BH (2005) Insulin-transferrin-selenium prevent human chondrocyte dedifferentiation and promote the formation of high quality tissue engineered human hyaline cartilage Eur Cell Mater 9:58-67; discussion 67
Han CL, Campbell GR, Campbell JH (2001) Circulating bone marrow cells can contribute to neointimal formation. J Vasc Res 38:113-119. https://doi.org/10.1159/ 000051038

Hellstrom M, Kalen M, Lindahl P, Abramsson A, Betsholtz C (1999) Role of PDGF-B and PDGFR-beta in recruitment of vascular smooth muscle cells and pericytes during embryonic blood vessel formation in the mouse. Development 126:3047-3055

Iwata $\mathrm{H}$ et al (2010) Bone marrow-derived cells contribute to vascular inflammation but do not differentiate into smooth muscle cell lineages. Circulation 122:2048-2057. https:// doi.org/10.1161/CIRCULATIONAHA.110.965202

Jackson CL, Reidy MA (1993) Basic fibroblast growth factor: its role in the control of smooth muscle cell migration. Am J Pathol 143:1024-1031

Jawien A, Bowen-Pope DF, Lindner V, Schwartz SM, Clowes AW (1992) Platelet-derived growth factor promotes smooth muscle migration and intimal thickening in a rat model of balloon angioplasty. J Clin Invest 89:507-511. https://doi.org/10.1172/JCI115613

Joo HJ et al (2014) Smooth muscle progenitor cells from peripheral blood promote the neovascularization of endothelial colony-forming cells. Biochem Biophys Res Commun 449:405-411. https://doi.org/10.1016/j.bbrc. 2014.05.061

Kang HS et al (2014) Smooth-muscle progenitor cells isolated from patients with moyamoya disease: novel experimental cell model. J Neurosurg 120:415-425. https://doi.org/10. 3171/2013.9.Jns131000

Kato S, Muraishi A, Miyamoto T, Fox JC (1998) Basic fibroblast growth factor regulates extracellular matrix and contractile protein expression independent of proliferation in vascular smooth muscle cells. In Vitro Cell Dev Biol Anim 34:341-346. https://doi.org/10.1007/s11626-998-0011-3

Lau CL (1993) Behavior of embryonic chick heart cells in culture. 1. Cellular responses to insulin-transferrin-selenium. Tissue Cell. 25:465-480

Libby P, O'Brien KV (1983) Culture of quiescent arterial smooth muscle cells in a defined serum-free medium. J Cell Physiol 115:217-223. https://doi.org/10.1002/jcp. 1041150217

Lin C, Yuan Y, Courtman DW (2016) Differentiation of Murine Bone Marrow-Derived Smooth Muscle Progenitor Cells Is Regulated by PDGF-BB and Collagen Plos One 11 doi:ARTN e015693510.1371/journal.pone.0156935

Lindner V, Reidy MA (1991) Proliferation of Smooth-Muscle Cells after Vascular Injury Is Inhibited by an Antibody against Basic Fibroblast Growth-Factor P Natl Acad Sci USA 88:3739-3743 doi:https://doi.org/10.1073/pnas.88.9. 3739

Majesky MW, Dong XR, Regan JN, Hoglund VJ (2011) Vascular smooth muscle progenitor cells building and repairing blood vessels. Circ Res 108:365-377. https://doi.org/ 10.1161/Circresaha.110.223800

Merkulova-Rainon T, Broqueres-You D, Kubis N, Silvestre JS, Levy BI (2012) Towards the therapeutic use of vascular smooth muscle progenitor cells. Cardiovasc Res 95:205-214. https://doi.org/10.1093/cvr/cvs097

Millette E, Rauch BH, Defawe O, Kenagy RD, Daum G, Clowes AW (2005) Platelet-derived growth factor-BB-induced 
human smooth muscle cell proliferation depends on basic FGF release and FGFR-1 activation. Circ Res 96:172-179. https://doi.org/10.1161/01.RES.0000154595.87608.db

Sata $M$ et al (2002) Hematopoietic stem cells differentiate into vascular cells that participate in the pathogenesis of atherosclerosis. Nat Med 8:403-409. https://doi.org/10. 1038/nm0402-403

Schroder K, Helmcke I, Palfi K, Krause KH, Busse R, Brandes RP (2007) Nox 1 mediates basic fibroblast growth factorinduced migration of vascular smooth muscle cells. Arterioscler Thromb Vasc Biol 27:1736-1743. https://doi.org/ 10.1161/ATVBAHA.107.142117

Shi N, Chen SY (2014) Mechanisms simultaneously regulate smooth muscle proliferation and differentiation J. Biomed Res 28:40-46. https://doi.org/10.7555/JBR.28.20130130

Shimizu K, Sugiyama S, Aikawa M, Fukumoto Y, Rabkin E, Libby P, Mitchell RN (2001) Host bone-marrow cells are a source of donor intimal smooth- muscle-like cells in murine aortic transplant arteriopathy. Nat Med 7:738-741. https://doi.org/10.1038/89121

Simper D, Stalboerger PG, Panetta CJ, Wang SH, Caplice NM (2002) Smooth muscle progenitor cells in human blood. Circulation 106:1199-1204. https://doi.org/10.1161/01. Cir.0000031525.61826.A8

Sugiyama S et al (2006) Characterization of smooth muscle-like cells in circulating human peripheral blood. Atherosclerosis 187:351-362. https://doi.org/10.1016/j. atherosclerosis.2005.09.014

van Oostrom O, Fledderus JO, de Kleijn D, Pasterkamp G, Verhaar MC (2009) Smooth muscle progenitor cells: friend or foe in vascular disease? Curr Stem Cell Res Ther 4:131-140

Waldbillig DK, Pang SC (1992) Differential Proliferation of Rat Aortic and Mesenteric Smooth-Muscle Cells in Culture. Histol Histopathol 7:199-207
Wang CH et al (2012) Surface markers of heterogeneous peripheral blood-derived smooth muscle progenitor cells. Arterioscler Thromb Vasc Biol 32:1875-1883. https://doi. org/10.1161/ATVBAHA.112.245852

Wang Q, Li HQ, Yao Y, Lu GH, Wang YH, Xia DJ, Zhou JY (2016) HB-EGF-promoted airway smooth muscle cells and their progenitor migration contribute to airway smooth muscle remodeling in asthmatic mouse. J Immunol 196:2361-2367. https://doi.org/10.4049/jimmunol. 1402126

Xie SZ et al (2008) Differentiation of smooth muscle progenitor cells in peripheral blood and its application in tissue engineered blood vessels. J Zhejiang Univ-Sc B 9:923-930. https://doi.org/10.1631/jzus.B0820257

Xie H et al (2014) PDGF-BB secreted by preosteoclasts induces angiogenesis during coupling with osteogenesis. Nat Med 20:1270-1278. https://doi.org/10.1038/nm.3668

Zernecke A et al (2005) SDF-1 alpha/CXCR4 axis is instrumental in neointimal hyperplasia and recruitment of smooth muscle progenitor cells. Circulation Res 96:784-791. https://doi.org/10.1161/01.Res.0000162100. 52009.38

Zhao Y, Lv WT, Piao HY, Chu XJ, Wang H (2014) Role of platelet-derived growth factor-BB (PDGF-BB) in human pulmonary artery smooth muscle cell proliferation. J Recept Sig Transd 34:254-260. https://doi.org/10.3109/ 10799893.2014.908915

Zoll J et al (2008) Role of human smooth muscle cell progenitors in atherosclerotic plaque development and composition. Cardiovasc Res 77:471-480. https://doi.org/10.1093/ cvr/cvm034

Publisher's Note Springer Nature remains neutral with regard to jurisdictional claims in published maps and institutional affiliations. 